\title{
Spatiotemporal Deformable Prototypes for Motion Anomaly Detection
}

\section{Robert Bensch}

bensch@cs.uni-freiburg.de

Thomas Brox

brox@cs.uni-freiburg.de

Olaf Ronneberger

ronneber@cs.uni-freiburg.de
Department of Computer Science and

BIOSS Centre for Biological Signalling Studies

University of Freiburg

Germany

http://lmb.informatik.uni-freiburg.de
Overview This paper presents an approach for motion-based anomaly detection from video in a new setting. The task is formulated as detecting instances of a well-defined motion pattern prototype, and localizing subtle anomalies in its context, see Fig. 1. To this end, the prototype pattern is detected and elastically registered against a test sample. This is quite different from the traditional anomaly detection setting for surveillance scenarios (e.g. [2]) where the absolute position is a valuable and predominant feature. While our setting is most related to reconstruction based approaches (e.g. [1]), it is different in that reconstruction is performed in a direct manner by registration. "Supertrajectories" based on hierarchical clustering of dense point trajectories serve as an efficient and robust representation of motion patterns. A statistical prototype model is learned from training sequences to define accepted variations in terms of spatiotemporal deformations and remaining deviations. We start with a robust detection, that allows for temporal shift and 3D spatial translation and rotation. An efficient hashing approach provides transformation hypotheses, that are refined by a spatiotemporal elastic registration of the deformable prototype. We propose a new method for elastic registration of $3 \mathrm{D}+$ time trajectory patterns that induces spatial elasticity from trajectory affinities. Finally, an unseen motion pattern is reconstructed by prototype placements, while subpatterns that remain poorly reconstructed are detected as abnormal. Compared to existing methods, that usually deal with 2D+time data, our method copes with 3D+time data. Fig. 1 illustrates our setting of anomaly detection with an example of juggling patterns. The method is evaluated on a new motion anomaly dataset (Figs. 1-2) and performs well in detecting subtle anomalies (Fig. 3). Moreover, we demonstrate the applicability to biological motion patterns (Fig. 4).

Results Our anomaly detection approach is basically different from most existing approaches. Accordingly, we found existing benchmarks to be inappropriate for demonstrating our method. We recorded a new motion anomaly dataset from persons juggling balls using a Kinect camera and generated 3D motion trajectories (Fig. 2). We compare against chaotic invariants (CI) for anomaly detection in crowded scenes [3]. Among the methods with code available, $\mathrm{CI}$ is the most related one as it provides invariance to position and magnitude via a bag of features approach. We show that our approach outperforms CI to its accurate modeling of the normal pattern variation (Fig. 3). Especially in the case of viewpoint variations compared to the training data, our approach clearly outperforms CI, see Fig. 3(a). Our method profits from its strong generalization capability due to spatial normalization by detection and elastic registration in 3D+time. Moreover, we demonstrate the relevance and general applicability in experiments on biological motion patterns (Fig. 4). We were able to quantify a significant difference between wild type (WT) and genetically modified embryos (MO) from global endodermal cell dynamics in zebrafish. In addition, we were able to find evidence for our hypothesis, that a time scaling might partially explain these differences. We will make the new motion anomaly dataset and the code publicly available.

[1] Y. Cong, J. Yuan, and J. Liu. Sparse reconstruction cost for abnormal event detection. In IEEE International Conference on Computer Vision and Pattern Recognition (CVPR), pages 3449-3456. IEEE, 2011.

[2] V. Mahadevan, Weixin Li, V. Bhalodia, and N. Vasconcelos. Anomaly detection in crowded scenes. In IEEE International Conference on Computer Vision and Pattern Recognition (CVPR), pages 1975-1981, June 2010.

[3] S. Wu, B. E. Moore, and M. Shah. Chaotic invariants of lagrangian particle trajectories for anomaly detection in crowded scenes. In IEEE International Conference on Computer Vision and Pattern Recognition (CVPR), pages 2054-2060, 2010.

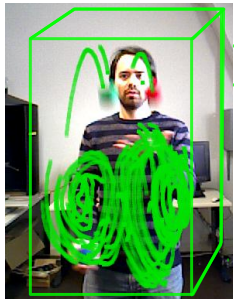

(a)

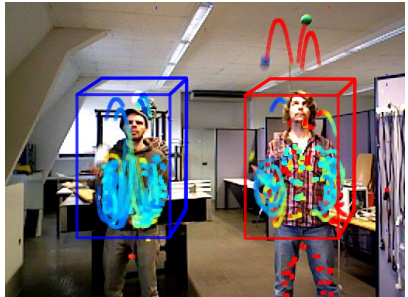

(b)

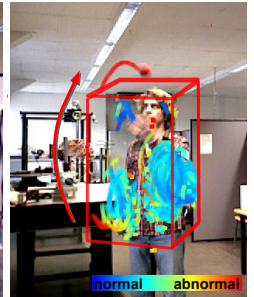

(c)
Figure 1: Motion anomaly detection in juggling patterns. (a) Motion pattern prototype. (b-c) Detection of local anomalies in context of prototype detections (bounding boxes). The anomaly score is plotted for supertrajectories from normal (blue) to abnormal (red).
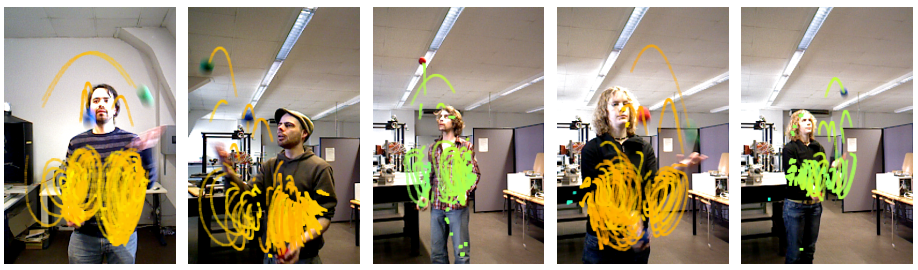

Figure 2: Juggling pattern test set. Examples of the same juggling pattern show possible variations due to different jugglers and viewpoints. Supertrajectories are plotted over a range of 45 frames $(1.5 \mathrm{sec})$, the color indicates depth obtained from Kinect camera.

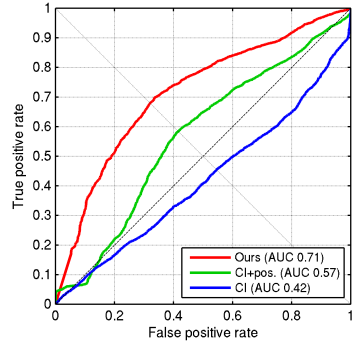

(a) Subset A

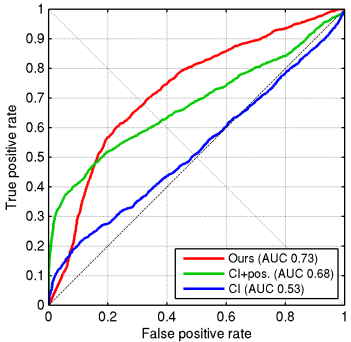

(b) Subset B (similar viewpoints)

Figure 3: Anomaly detection ROC curves. Comparison of our method (red) and the chaotic invariants (CI) [3] (green and blue) for the two subsets A and B. The average performance of all methods is shown.

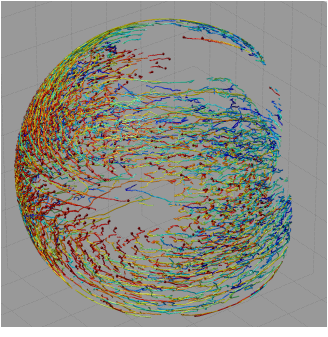

(a) Wild type (WT)

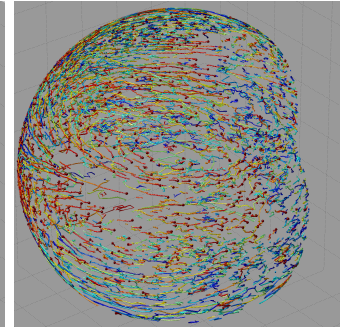

(b) cxcr4a morphant (MO)

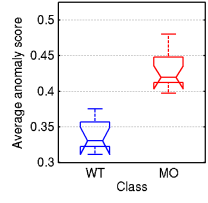

(c) WT vs. MO

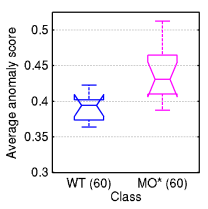

(d) WT vs. MO*

Figure 4: Anomaly detection in biological motion patterns of cell dynamics in the early development of zebrafish embryos. (a-b) Example motion patterns for each group. Trajectories are rendered in 3D with time colourcoded from blue to red. (c-d) Boxplots of global anomaly scores. (c) Significant difference between WT and MO patterns. (d) Time scaling of MO patterns (MO*) partially compensates the differences. 